TITLE:

\title{
Computing the distance to uncontrollability via LMIs: Lower bound computation with exactness verification
}

\author{
AUTHOR(S):
}

Ebihara, Yoshio

\section{CITATION:}

Ebihara, Yoshio. Computing the distance to uncontrollability via LMIs: Lower bound computation with exactness verification. Systems \& Control Letters 2008, 57(9): 763-771

\section{ISSUE DATE:}

2008-09

URL:

http://hdl.handle.net/2433/85170

\section{RIGHT:}

Copyright (C) 2008 Elsevier B.V.; この論文は出版社版でありません。引 用の際には出版社版をご確認ご利用ください。; This is not the published version. Please cite only the published version. 


\title{
Computing the Distance to Uncontrollability via LMIs: Lower Bound Computation with Exactness Verification*
}

\author{
Yoshio Ebihara \\ Department of Electrical Engineering, Kyoto University, \\ Kyotodaigaku-Katsura, Nishikyo-ku, Kyoto 615-8510, Japan. \\ Tel(Fax): 81-75-383-2252, e-mail:ebihara@kuee.kyoto-u.ac.jp.
}

November 1, 2007

\begin{abstract}
In this paper, we consider the problem to compute the distance to uncontrollability (DTUC) of a given controllable pair $A \in \mathbf{C}^{n \times n}$ and $B \in \mathbf{C}^{n \times m}$. It is known that this problem is equivalent to computing the minimum of the smallest singular value of $\left[\begin{array}{lll}A-z I & B\end{array}\right]$ over $z \in \mathbf{C}$. With this fact, $\mathrm{Gu}$ et al. proposed an algorithm that correctly estimates the DTUC at a computation cost $O\left(n^{4}\right)$. From the viewpoints of linear control system theory, on the other hand, this problem can be regarded as a special case of the structured singular value computation problems and thus it is expected that we can establish an alternative LMI-based algorithm. In fact, this paper first shows that we can compute a lower bound of the DTUC by simply applying the existing techniques to solve robust LMIs. Moreover, we show via convex duality theory that this lower bound can be characterized by a very concise dual SDP. In particular, this dual SDP enables us to derive a condition on the dual variable under which the computed lower bound surely coincides with the exact DTUC. On the other hand, in the second part of the paper, we consider the problem to compute the similarity transformation matrix $T$ that maximizes the lower bound of the DTUC of $\left(T^{-1} A T, T^{-1} B\right)$. We clarify that this problem can be reduced to a generalized eigenvalue problem and thus solved efficiently. In view of the correlation between the DTUC and the numerical difficulties of the associated pole placement problem, this computation of the transformation matrix would lead to an effective and efficient conditioning of the pole placement problem for the pair $(A, B)$.
\end{abstract}

\section{Introduction}

Let us consider the linear system described by

$$
\dot{x}(t)=A x(t)+B u(t), \quad A \in \mathbf{R}^{n \times n}, \quad B \in \mathbf{R}^{n \times m} .
$$

Here, $x \in \mathbf{R}^{n}$ is the state vector and $u \in \mathbf{R}^{m}$ is the control input vector. As is wellknown, this system is said to be controllable if, for given any initial and final states $x(0)$ and $x(T)$, there exists $u(t)(0 \leq t \leq T)$ that drives the state from $x(0)$ to $x(T)$. The controllability of the system (1), or say, the controllability of the matrix pair $(A, B)$, can

*This work is supported in part by the Ministry of Education, Culture, Sports, Science and Technology of Japan under Grant-in-Aid for Young Scientists (B), 18760319. 
be tested algebraically via the Popov-Belevitch-Hautus $(\mathrm{PBH})$ test [26]. We can also check the controllability via the positivity of the associated controllability gramian [26].

For the controllable pair $(A, B)$, its distance to uncontrollability (DTUC) was firstly introduced in [19] and can be characterized as follows [8]:

$$
\varepsilon^{\star}=\min _{z \in \mathbf{C}} \sigma_{\min }([A-z I B]) .
$$

Here, $\sigma_{\min }(\cdot)$ denotes the smallest singular value. The form $(2)$ conforms to the PBH test, which claims that the pair $(A, B)$ is controllable iff $\operatorname{rank}([A-z I B])=n(\forall z \in \mathbf{C})$.

The computation of the DTUC is motivated by the following facts:

- The classical controllability tests do not provide any means to detect whether the control system is nearly uncontrollable, which is equally troublesome in practice [9].

- The DTUC can be used as a measure to estimate whether the pole placement problem for the pair $(A, B)$ is well- or ill-conditioned. More precisely, the pole placement problem is likely to be ill-conditioned if the DTUC is small $[13,16]$.

In view of these facts, intensive research efforts have been made for the computation of the DTUC. As clearly shown in (2), the DTUC can be computed by solving a global optimization problem in two real variables. In general, this is a non-convex optimization problem and thus not tractable [9]. Recently, however, linear algebra algorithms that correctly estimate the distance at a cost of polynomial in $n$ have been developed $[9,4,10]$.

From the viewpoints of linear control system theory, on the other hand, it can be seen from (2) that the DTUC computation can be regarded as a special case of the structured singular value (SSV) computation [18]. Since linear matrix inequality (LMI) is surely powerful for the SSV computation, it is expected that we can construct alternative LMIbased algorithms to correctly compute the DTUC. However, as clearly stated in [4], it does not seem to be known whether the Gu's algorithm could be replaced by an LMI-based one and this fact motivates us to compute the DTUC via LMIs in this paper.

It should be noted that our primary concern is not to pursue computationally demanding LMI-based algorithms but to shed more insight to the problem from convex duality theory. To this end, we first reveal that a lower bound of the DTUC can readily be computed by applying existing techniques to deal with robust LMIs [2, 12, 15, 17]. Next we show via convex duality theory [1] that this lower bound can be characterized by a very concise dual SDP. This dual SDP enables us to derive a rank condition on the dual variable under which the computed lower bound surely coincides with the exact DTUC. We also show that, if the suggested rank condition is satisfied, one of the global optimizers $z^{\star} \in \mathbf{C}$ for (2) can be obtained by simply computing the eigenvalues of a matrix constructed from the optimal dual variable. This rank condition works fine in practice, and we will show thorough numerical examples that we can obtain exactness certificate even for those problems where the common rank-one exactness principle fails.

On the other hand, in the second part of the paper, we consider the problem to compute the similarity transformation matrix $T$ that maximizes the lower bound of the DTUC of $\left(T^{-1} A T, T^{-1} B\right)$. In particular, we will show that this problem can be reduced to generalized eigenvalue problem and thus solved efficiently. In view of the correlation between the DTUC and the numerical difficulties of the associated pole placement problem $[13,16]$, this computation of the similarity transformation matrix would lead to an 
effective and efficient conditioning of the pole placement problem for the pair $(A, B)$. This illustrates in part the usefulness of the proposed LMI-based methodology over the existing linear algebra algorithms $[9,4,10]$.

We use the following notations in this paper. The symbols $\mathbf{H}_{n}, \mathbf{P}_{n}$ and $\mathbf{S}_{n}$ denote respectively the set of $n \times n$ Hermitian matrices, positive-semidefinite, and skew-Hermitian matrices. For $V \in \mathbf{H}_{n}$, we denote its smallest eigenvalue by $\lambda_{\min }(V)$.

\section{Problem Formulation and Perspectives of the LMI-based Results}

\subsection{Problem Formulation}

For given $A \in \mathbf{C}^{n \times n}$ and $B \in \mathbf{C}^{n \times m}$, our primary concern is to compute the DTUC given by (2). By following [4, 7], however, we proceed our discussion under slightly more general setting. Namely, for given $P, Q \in \mathbf{C}^{n \times(n+m)}$, we consider the problem to compute

$$
\varepsilon^{\star}:=\min _{z \in \mathbf{C}} \sigma_{\min }(P+z Q)
$$

or equivalently,

$$
\varepsilon^{\star}:=\min _{(r, \theta) \in \mathbf{R} \times(-\pi, \pi]} \sigma_{\min }\left(P+r e^{j \theta} Q\right) .
$$

We assume that rank $Q=n$. The problem to compute the DTUC can be seen as a special case of (3) where $P=[A B]$ and $Q=\left[-I_{n} 0_{n, m}\right]$.

\subsection{Perspectives of the LMI-based Results}

Since $\lim _{|z| \rightarrow \infty} \sigma_{\min }(P+z Q) \rightarrow \infty$ holds under our assumption that $Q$ is of full-row rank, and since $\sigma_{\min }(P+z Q)$ take its value $\sigma_{\min }(P)$ at $z=0$, it is obvious that there exists $\gamma_{l}=\gamma_{l}(P, Q)>0$ such that

$$
\varepsilon^{\star}=\min _{|z| \leq \gamma_{l}} \sigma_{\min }(P+z Q) .
$$

In the next section, we give a detailed analysis on how to determine the value $\gamma_{l}>0$ reasonably. Furthermore, from the definition of the smallest singular value, we can rewrite (5) as

$$
\varepsilon^{\star}=\max \varepsilon \text { subject to } \quad(P+z Q)(P+z Q)^{*}-\varepsilon^{2} I \geq 0 \quad \forall|z| \leq \gamma_{l}
$$

or equivalently,

$$
\varepsilon^{\star}=\max \varepsilon \text { subject to }\left[\begin{array}{c}
I_{n} \\
z I_{n}
\end{array}\right]^{*}\left[\begin{array}{cc}
P P^{*}-\varepsilon^{2} I & Q P^{*} \\
P Q^{*} & Q Q^{*}
\end{array}\right]\left[\begin{array}{c}
I_{n} \\
z I_{n}
\end{array}\right] \geq 0 \quad \forall|z| \leq \gamma_{l} .
$$

In relation to (6), let us consider the following two conditions to be verified:

$$
\left[\begin{array}{c}
I_{n} \\
z I_{n}
\end{array}\right]^{*} \Phi\left[\begin{array}{c}
I_{n} \\
z I_{n}
\end{array}\right] \geq 0 \quad \forall|z|=\gamma_{l}
$$




$$
\left[\begin{array}{c}
I_{n} \\
z I_{n}
\end{array}\right]^{*} \Psi\left[\begin{array}{c}
I_{n} \\
z I_{n}
\end{array}\right]>0 \quad \forall|z| \leq \gamma_{l}
$$

Here, $\Phi, \Psi \in \mathbf{H}_{2 n}$ are given matrices with $\Psi \geq 0$. It is known that these conditions form important basis for linear system analysis $[12,14,15,23,6]$. For example, the condition (7) is strongly related to the celebrated KYP lemma $[15,20]$ while the condition (8) is related to the basic properties of linear systems such as stability and stabilizability [6]. In particular, it has been shown that the problems to verify these conditions can be reduced into LMI feasibility problems in an exact fashion $[12,15,23,6]$.

As clearly seen, the optimization problem (6) does not fall into the category of (7) or (8). To the best of the author's knowledge, exact LMIs for (7) with $\forall|z|=\gamma_{l}$ replaced by $\forall|z| \leq \gamma_{l}$ or for (8) without imposing $\Psi \geq 0$ are not known in the literature. Thus, simple application of the existing LMI-related results does not allow us to solve the problem to compute the DTUC exactly.

\section{Lower Bounds Computation}

\subsection{Reduction to a Single Variable Optimization Problem over a Compact Set}

Let us consider the definition of $\varepsilon^{\star}$ given by (4). In this optimization problem, it is almost apparent that we can bound the radius $r$ as we have stated around (5). Indeed, we can readily obtain the next result.

Proposition 1 The quantity $\varepsilon^{\star}$ in (4) can also be characterized as

$$
\varepsilon^{\star}=\min _{(r, \theta) \in\left[-\gamma_{l}, \gamma_{l}\right] \times(-\pi, \pi]} \sigma_{\min }\left(P+r e^{j \theta} Q\right)
$$

where

$$
\gamma_{l}:=\sqrt{\frac{\sigma_{\min }(P)^{2}+1}{\lambda_{\min }\left(Q\left(I+P^{*} P\right)^{-1} Q^{*}\right)}} .
$$

Proof: We first note that, since the matrix $Q$ is of full row-rank from our underlying assumption, $Q\left(I+P^{*} P\right)^{-1} Q^{*}>0$ and thus $\gamma_{l}>0$ is well-defined.

To prove the assertion, it suffices to show that the optimizer $r$ in (4) cannot lie on $|r|>\gamma_{l}$. To this end, we note from (10) that the following inequality holds:

$$
r^{2}>\frac{\sigma_{\min }(P)^{2}+1}{\lambda_{\min }\left(Q Q^{*}-Q P^{*}\left(P P^{*}+I\right)^{-1} P Q^{*}\right)} \quad \forall|r|>\gamma_{l} .
$$

Here, we applied the matrix inversion formula [26] to the term $\left(I+P^{*} P\right)^{-1}$ in (10). It follows that

$$
r^{2}\left(Q Q^{*}-Q P^{*}\left(P P^{*}+I\right)^{-1} P Q^{*}\right)-\left(\sigma_{\min }(P)^{2}+1\right) I>0 \quad \forall|r|>\gamma_{l} .
$$

From the Schur Complements arguments, we obtain

$$
\left[\begin{array}{cc}
r^{2} Q Q^{*}-\sigma_{\min }(P)^{2} I-I & r Q P^{*} \\
r P Q^{*} & P P^{*}+I
\end{array}\right]>0 \quad \forall|r|>\gamma_{l} .
$$


By pre-multiplying $\left[\begin{array}{ll}I & e^{-j \theta} I\end{array}\right]$ and post-multiplying $\left[\begin{array}{ll}I & e^{-j \theta} I\end{array}\right]^{*}$ to the this inequality, we have

$$
\left(P+r e^{j \theta} Q\right)\left(P+r e^{j \theta} Q\right)^{*}>\sigma_{\min }(P)^{2} I, \quad \forall|r|>\gamma_{l}, \quad \forall \theta \in(-\pi, \pi] .
$$

This implies $\varepsilon_{\gamma_{l}}^{\star}>\sigma_{\min }(P)$ where

$$
\varepsilon_{\gamma_{l}}^{\star}:=\min _{|r|>\gamma_{l}, \theta \in(-\pi, \pi]} \sigma_{\min }\left(P+r e^{j \theta} Q\right) .
$$

On the other hand, it is also obvious that $\varepsilon^{\star} \leq \sigma_{\min }(P)$ since the quantity $\sigma_{\min }(P)$ corresponds to the one we evaluate $\sigma_{\min }\left(P+r e^{j \theta} Q\right)$ at $r=0$. By noting these facts as well as $0 \in\left[-\gamma_{l}, \gamma_{l}\right]$, the proof is completed.

In Proposition 1, we have derived an explicit bound on the radius $r$. We note that, even if the bound $\gamma_{l}$ might not be tight, the fact that we can bound the radius $r$ plays an important role to derive the main result of this paper in Section 4 (see the discussion around (19) in the proof of Theorem 1).

We next remove the dependence on $\theta$ of (9). To this end, we first note that (9) can be rewritten, equivalently, as

$$
\begin{aligned}
\varepsilon^{\star}=\max & \varepsilon \text { subject to } \\
& {\left[\begin{array}{c}
I \\
e^{j \theta}
\end{array}\right]^{*}\left[\begin{array}{cc}
r^{2} Q Q^{*}-\varepsilon^{2} I & r Q P^{*} \\
r P Q^{*} & P P^{*}
\end{array}\right]\left[\begin{array}{c}
I \\
e^{j \theta}
\end{array}\right] \geq 0 \quad \forall(r, \theta) \in\left[-\gamma_{l}, \gamma_{l}\right] \times(-\pi, \pi] . }
\end{aligned}
$$

Then, for each fixed $r \in\left[-\gamma_{l}, \gamma_{l}\right]$, we see from the KYP lemma $[15,20]$ that

$$
\left[\begin{array}{c}
I \\
e^{j \theta}
\end{array}\right]^{*}\left[\begin{array}{cc}
r^{2} Q Q^{*}-\varepsilon^{2} I & r Q P^{*} \\
r P Q^{*} & P P^{*}
\end{array}\right]\left[\begin{array}{c}
I \\
e^{j \theta}
\end{array}\right] \geq 0 \quad \forall \theta \in(-\pi, \pi]
$$

holds if and only if there exists $X(r) \in \mathbf{H}_{n}$ such that $\Gamma(P, Q, r, X(r), \varepsilon) \geq 0$, where

$$
\Gamma(P, Q, r, X(r), \varepsilon):=\left[\begin{array}{cc}
r^{2} Q Q^{*}-\varepsilon^{2} I-X(r) & r Q P^{*} \\
r P Q^{*} & P P^{*}+X(r)
\end{array}\right] .
$$

This observation leads us to the next result, which forms an important basis of our study.

Proposition 2 The quantity $\varepsilon^{\star}$ in (4) can also be characterized as

$$
\varepsilon^{\star}=\max _{X(r) \in \mathbf{H}_{n}} \varepsilon \text { subject to } \quad \Gamma(P, Q, r, X(r), \varepsilon) \geq 0 \quad \forall r \in\left[-\gamma_{l}, \gamma_{l}\right]
$$

Here we have introduced $X(r): \mathbf{R} \rightarrow \mathbf{H}_{n}$ so that we can eliminate the dependence on the parameter $\theta$ of (12). This type of matrix is often called a parameter-dependent multiplier (PDM) [5]. In particular, the idea to reduce the number of parameters by introducing PDMs via the KYP lemma was first introduced in $[2,17]$. Originally, the KYP lemma has been used to convert frequency domain inequalities of linear systems into LMIs [3]. Recently, however, this lemma receives renewed interest to deal with robust LMI problems; see, e.g., [2, 15, 17, 24]. 


\subsection{Lower Bounds Computation via Finite Power Series Approximation of $X(r)$}

In Proposition 2, we have reduced the DTUC computation problem into an optimization problem of single variable over a compact set. However, the resulting problem is still intractable since we have to seek for the desired $X(r)$ over an infinite-dimensional function space. To get around this difficulty, in this subsection, we consider finite degree polynomial approximation of $X(r)$ and provide a numerically tractable way to compute the lower bounds of the DTUC.

For given $N$, let us define $X_{N}(r):=\sum_{i=0}^{N} r^{i} X_{i}$ where $X_{i} \in \mathbf{H}_{n}(i=0, \cdots, N)$ are decision variables to be determined through optimization. We further define

$$
\varepsilon_{N}^{\star}:=\max _{X_{N}(r) \in \mathbf{H}_{n}} \varepsilon \text { subject to } \quad \Gamma\left(P, Q, r, X_{N}(r), \varepsilon\right) \geq 0 \quad \forall r \in\left[-\gamma_{l}, \gamma_{l}\right] .
$$

Then, it is apparent that $\varepsilon^{\star} \geq \varepsilon_{j}^{\star} \geq \varepsilon_{k}^{\star}(j \geq k)$. Once we have restricted our attention on $X_{N}(r)$, we can reduce the problem to compute the corresponding lower bound $\varepsilon_{N}^{\star}$ defined in (14) into an SDP in an exact fashion (see, e.g., $[12,15])$. For example, in the case where $N=2$, we can readily obtain the next result.

Proposition 3 The quantity $\varepsilon_{2}^{\star}$ can be computed via SDP:

$$
\begin{aligned}
& \varepsilon_{2}^{\star}=\max _{X_{0}, X_{1}, X_{2}, D, G} \varepsilon \quad \text { subject to } \\
& \begin{array}{l}
{\left[\begin{array}{cc:cc}
-\varepsilon^{2} I-X_{0} & 0 & -\frac{1}{2} X_{1} & Q P^{*} \\
0 & P P^{*}+X_{0} & 0 & \frac{1}{2} X_{1} \\
\hdashline-\frac{1}{2} X_{1} & 0 & Q Q^{*}-X_{2} & 0 \\
P Q^{*} & \frac{1}{2} X_{1} & 0 & X_{2}
\end{array}\right]+\left[\begin{array}{cc}
-\gamma_{l}^{2} D & G \\
G^{*} & D
\end{array}\right] \geq 0,} \\
X_{0}, X_{1}, X_{2} \in \mathbf{H}_{n}, \quad D \in \mathbf{P}_{2 n}, \quad G \in \mathbf{S}_{2 n} .
\end{array}
\end{aligned}
$$

By solving the $\operatorname{SDP}(15)$, we can obtain the lower bound $\varepsilon_{2}^{\star}$ for $\varepsilon^{\star}$ as well as $X_{0}, X_{1}$ and $X_{2}$. Using these matrices $X_{0}, X_{1}$ and $X_{2}$, in [7], an efficient method to compute an upper bound of $\varepsilon^{\star}$ is also suggested.

\section{Exactness Verification of Lower Bounds}

It was shown in [7] that the aforementioned lower bound $\varepsilon_{2}^{\star}$ is very close to the exact DTUC in all tested numerical examples. However, since the computation of $\varepsilon_{2}^{\star}$ is based on the second-order approximation of $X(r)$ in (13), we cannot say anything theoretically on the exactness of $\varepsilon_{2}^{\star}$ at this stage.

On the other hand, in order to verify the exactness of the LMI relaxation approaches in other context of linear system analysis and synthesis, it is surely effective to consider the dual problem and examine the structure of the dual variable $[11,14,15,21,22,23,6]$. This often leads to rigorous theoretical proofs for the exactness of LMIs at hand $[11,14,15,6]$, and even for those hard problems that do not allow us to conclude the exactness, we can obtain viable condition for the exactness verification [21, 22, 23].

Following these ideas, we first consider the characterization of $\varepsilon_{2}^{\star}$ in (15) via dual SDP. 
Lemma 1 The quantity $\varepsilon_{2}^{\star}$ can also be characterized by the dual SDP:

$$
\begin{aligned}
& \varepsilon_{2}^{\star 2}=\min _{H} \operatorname{trace}\left(\left[\begin{array}{ll}
P P^{*} & P Q^{*} \\
Q P^{*} & Q Q^{*}
\end{array}\right] H\right) \text { subject to } \\
& H:=\left[\begin{array}{ll}
H_{11} & H_{12} \\
H_{12}^{*} & H_{22}
\end{array}\right] \geq 0 \\
& \widehat{H}:=\left[\begin{array}{ll}
H_{11} & H_{12}^{*} \\
H_{12} & H_{22}
\end{array}\right] \geq 0 \\
& \gamma_{l}^{2} H_{11} \geq H_{22} \\
& \operatorname{trace}\left(H_{11}\right)=1 .
\end{aligned}
$$

Proof: See the appendix section.

Lemma 1 shows that we can compute the lower bound $\varepsilon_{2}^{\star}$ by solving the dual SDP with $2 n^{2}+n$ scalar variables, whereas the primal SDP (15) involves $\left(11 n^{2}+3 n\right) / 2$ scalar variables. The total size of LMIs are $5 n$ and $6 n$, respectively. From [25], the computational complexity to solve an SDP is represented by $O\left(K^{2} R^{2.5}+R^{3.5}\right)$, where $K$ denotes the number of scalar veriables involved and $R$ the size of the underlying LMI. In view of these facts, it is clear that we have achieved considerable reduction of the associated computational burden by Lemma 1 .

In addition, the simplification into (16) enables us to obtain the next result, which works effectively in practice for the exactness verification of the lower bound $\varepsilon_{2}^{\star}$.

Theorem 1 Let us denote the full-rank factorization of the optimal $H$ and $\widehat{H}$ in Lemma 1 by

$$
H=\left[\begin{array}{l}
H_{1} \\
H_{2}
\end{array}\right]\left[\begin{array}{l}
H_{1} \\
H_{2}
\end{array}\right]^{*}, \quad \widehat{H}=\left[\begin{array}{c}
\widehat{H}_{1} \\
\widehat{H}_{2}
\end{array}\right]\left[\begin{array}{l}
\widehat{H}_{1} \\
\widehat{H}_{2}
\end{array}\right]^{*} .
$$

If both $H_{1}$ and $\widehat{H}_{1}$ in (17) are full column-rank, we have $\varepsilon_{2}^{\star}=\varepsilon^{\star}$.

Proof: Since $\varepsilon_{2}^{\star} \leq \varepsilon^{\star}$ holds, it suffices to prove $\varepsilon_{2}^{\star} \geq \varepsilon^{\star}$ if both $H_{1}$ and $\widehat{H}_{1}$ in (17) are full column-rank. To this end, we first note from the structure of $H$ and $\widehat{H}$ in (16b) and (16c) that

$$
H_{1} H_{1}^{*}=\widehat{H}_{1} \widehat{H}_{1}^{*} \text {. }
$$

Since both $H_{1}$ and $\widehat{H}_{1}$ are of full-column rank from our underlying assumption, the above equality implies that the column-size of $H_{1}$ and $\widehat{H}_{1}$ are the same, which is denoted by $k$ in the sequel. Then, from (18) and [20, Lemma 3], we see that there exists a unitary matrix $\Omega \in \mathbf{C}^{k \times k}$ such that $\widehat{H}_{1}=H_{1} \Omega$. Thus, the matrix $\widehat{H}$ can be rewritten in the following form:

$$
\widehat{H}=\left[\begin{array}{c}
H_{1} \\
\bar{H}_{2}
\end{array}\right]\left[\begin{array}{c}
H_{1} \\
\bar{H}_{2}
\end{array}\right]^{*}\left(\bar{H}_{2}:=\widehat{H}_{2} \Omega^{*}\right)
$$


On the other hand, from (16b), (16c) and (16d), we have $\gamma_{l}^{2} H_{1} H_{1}^{*} \geq H_{2} H_{2}^{*}$ and $\gamma_{l}^{2} H_{1} H_{1}^{*} \geq$ $\bar{H}_{2} \bar{H}_{2}^{*}$. Hence, again from [20, Lemma 3], there exist matrices $V, W \in \mathbf{C}^{k \times k}$ with $\|V\| \leq \gamma_{l}$ and $\|W\| \leq \gamma_{l}$ such that

$$
H_{2}=H_{1} V, \quad \bar{H}_{2}=H_{1} W
$$

It follows that we can represent $H$ and $\widehat{H}$ in the following form:

$$
H=\left[\begin{array}{cc}
H_{1} H_{1}^{*} & H_{1} V^{*} H_{1}^{*} \\
H_{1} V H_{1}^{*} & H_{1} V V^{*} H_{1}^{*}
\end{array}\right], \quad \widehat{H}=\left[\begin{array}{cc}
H_{1} H_{1}^{*} & H_{1} W^{*} H_{1}^{*} \\
H_{1} W H_{1}^{*} & H_{1} W W^{*} H_{1}^{*}
\end{array}\right] .
$$

Since $H_{1}$ is full-column rank, we see from (16b), (16c) and (20) that $V=W^{*}$ and $V V^{*}=W W^{*}$ hold. This implies $V V^{*}=V^{*} V$. Namely, the matrix $V$ is a normal matrix. Therefore there exists a unitary matrix $U \in \mathbf{C}^{k \times k}$ such that

$V=U \Lambda_{V} U^{*}$

Here, $\Lambda_{V}$ is a diagonal matrix whose diagonal entries $\lambda_{V, 1} \cdots \lambda_{V, k}$ satisfy

$$
\lambda_{V, i}=r_{i} e^{j \theta_{i}}, r_{i} \in \mathbf{R},\left|r_{i}\right| \leq \gamma_{l}, \quad \theta_{i} \in(-\pi, \pi] \quad(i=1, \cdots, k) .
$$

We now move onto the final stage of the proof. With the unitary matrix $U$ in (21), let us denote $H_{1} U=\left[h_{1} \cdots, h_{k}\right]$. Then, we have from (19), (21) and (22) that

$$
H=\left[\begin{array}{c}
H_{1} \\
H_{2}
\end{array}\right]\left[\begin{array}{c}
H_{1} \\
H_{2}
\end{array}\right]^{*}=\sum_{i=1}^{k}\left[\begin{array}{c}
h_{i} \\
h_{i} r_{i} e^{j \theta i}
\end{array}\right]\left[\begin{array}{c}
h_{i} \\
h_{i} r_{i} e^{j \theta i}
\end{array}\right]^{*} .
$$

From this factorization, (16a) and (16e), we see that there exists at lease one index $j(1 \leq j \leq k)$ such that

$$
\operatorname{trace}\left(\left[\begin{array}{cc}
-\varepsilon_{2}^{\star 2} I+P P^{*} & P Q^{*} \\
Q P^{*} & Q Q^{*}
\end{array}\right]\left[\begin{array}{c}
h_{j} \\
h_{j} r_{j} e^{j \theta_{j}}
\end{array}\right]\left[\begin{array}{c}
h_{j} \\
h_{j} r_{j} e^{j \theta_{j}}
\end{array}\right]^{*}\right) \leq 0
$$

or equivalently, $h_{j}^{*}\left(-\varepsilon_{2}^{\star 2} I+\left(P+r_{j} e^{-j \theta_{j}} Q\right)\left(P+r_{j} e^{-j \theta_{j}} Q\right)^{*}\right) h_{j} \leq 0$. This implies $\varepsilon_{2}^{\star} \geq$ $\sigma_{\min }\left(P+r_{j} e^{-j \theta_{j}} Q\right) \geq \varepsilon^{\star}$ and thus we complete the proof.

Theorem 1 can be used in the following way. By solving the SDP (16), we can obtain $\varepsilon_{2}^{\star}$ as well as the optimal $H$ and $\widehat{H}$. At this stage, we cannot say anything on the strictness of $\varepsilon_{2}^{\star}$. However, if the matrices $H_{1}$ and $\widehat{H}_{1}$ resulting from the full-rank factorization of $H$ and $\widehat{H}$ as in (17) are both full-column rank, we can conclude that $\varepsilon_{2}^{\star}=\varepsilon^{\star}$ surely holds. We note that the numerical determination of rank can be an ill-conditioned calculation, though this issue does not arise in our all tested numerical experiments.

From the proof of Theorem 1, we see that if the assumption in Theorem 1 is satisfied, then at least one of the eigenvalues of $V^{*}=W$ is the global optimizer. From (19), it can be seen that the matrix $W$ satisfies $\widehat{H}_{2}=\widehat{H}_{1} \Omega^{*} W \Omega$ and hence the eigenvalues of $W$ coincides with those of $X:=\left(\widehat{H}_{1}^{*} \widehat{H}_{1}\right)^{-1} \widehat{H}_{1}^{*} \widehat{H}_{2}$, which can readily be constructed from (17). This is stated formally in the next corollary. 
Corollary 1 Let us denote the full-rank factorization of the optimal $H$ and $\widehat{H}$ in Lemma 1 by (17) and suppose both $H_{1}$ and $\widehat{H}_{1}$ are full column-rank. We further define a matrix $X$ from (17) as follows:

$X=\left(\widehat{H}_{1}^{*} \widehat{H}_{1}\right)^{-1} \widehat{H}_{1}^{*} \widehat{H}_{2}$.

Then, at least one of the eigenvalues of the matrix $X$ coincides with the global optimizer $z^{\star}$ of (3).

On the other hand, if both the matrices $H$ and $\widehat{H}$ in Lemma 1 are rank-one, it is apparent that the assumption in Theorem 1 is automatically satisfied. Hence, we see that the next corollary holds.

Corollary 2 If the optimal $H$ and $\widehat{H}$ in Lemma 1 are both rank-one, then $\varepsilon_{2}^{\star}=\varepsilon^{\star}$. In particular, one of the global optimizer $z^{\star}$ of (3) can be computed from (17) via

$z^{\star}=\left(\widehat{H}_{1}^{*} \widehat{H}_{1}\right)^{-1} \widehat{H}_{1}^{*} \widehat{H}_{2}$.

Remark 1 In the preceding studies for the exactness verifications of LMI relaxations, so called rank-one exactness principle is derived (see related discussions in [11, 22, 23] and references therein). In our context, this requires both $H$ and $\widehat{H}$ in Lemma 1 are rankone as in Corollary 2. Namely, the rank condition in Theorem 1 is more general than this common rank-one exactness principle, and this generalization is quite important to extract the optimizers as shown in numerical examples given below.

It should be noted that the recent paper [24] includes exactness results that go beyond [22, 23]. Roughly speaking, the approach in [24] is quite general and can be applied to whole variety of robust LMI problems. On the other hand, the approach in the present paper is very specific; the exactness test in Theorem 1 has been derived successfully by relying on the particular structure of the DTUC computation problem.

Example 1: Let us illustrate the effectiveness of Theorem 1 as well as Corollary 1 through numerical examples ${ }^{1}$. To this end, we solve (16) for the matrix data found at [27].

Firstly, we solved the SDP (16) for the pair $(A, B)$ named "Markov_Chain104," where the size of $(A, B)$ are $A \in \mathbf{R}^{10 \times 10}$ and $B \in \mathbf{R}^{10 \times 4}$. It turned out that $\varepsilon_{2}^{\star}=0.0769$. The computation time was 0.41 [sec].

To examine the exactness of the lower bound $\varepsilon_{2}^{\star}$, we next check the rank of the resulting optimal variables $H$ and $\widehat{H}$ in (16). It turns out that the largest singular value of $H$ is 1.0638 and other singular values are of less than $10^{-8}$. Similar comments apply also to the singular values of $\widehat{H}$ (in fact, the matrix $\widehat{H}$ is very close to $H$ in this case). Thus both $H$ and $\widehat{H}$ can be regarded to be rank-one and hence from Corollary 2, we can concluded that $\varepsilon_{2}^{\star}=\varepsilon^{\star}$. The global optimizer computed from (24) is $z^{\star}=-0.2526$. In Fig. 1, we show the contour plots of $\log _{10}\left(\sigma_{\min }([A-z I B])\right)$ and the location of $z^{\star}$.

Next we solved the SDP (16) for the pair $(A, B)$ named "Grcar104," where the size of these matrices are again $A \in \mathbf{R}^{10 \times 10}$ and $B \in \mathbf{R}^{10 \times 4}$. The resulting value was $\varepsilon_{2}^{\star}=0.4418$ and the computation time 0.43 [sec].

\footnotetext{
${ }^{1}$ In this paper, every LMI-related computation is carried out with SeDuMi [25] and MATLAB R2006a, on PC with CPU Pentium IV 3.6 GHz.
} 
By examining the resulting optimal variables $H$ and $\widehat{H}$, it turns out that both $H$ and $\widehat{H}$ are of rank-two. Hence, in contrast with the preceding example, we cannot obtain exactness certificate via Corollary 2. However, by carrying out the full-rank factorization of $H$ and $\widehat{H}$ as in (17), we confirmed that both $H_{1}$ and $\widehat{H}_{1}$ are of full-column rank. Thus, from Theorem 1, we can conclude that $\varepsilon_{2}^{\star}$ surely coincides with the global optimum $\varepsilon^{\star}$.

Since both $H_{1}$ and $\widehat{H}_{1}$ are of full-column rank in (17), we can compute the global optimizer $z^{\star}$ from Corollary 1. The eigenvalues of $X$ in (23) are computed as $1.3842 \pm$ $0.9738 j$. Since these eigenvalues form a complex conjugate pair, it can be seen that both of these eigenvalues are the global optimizers. In Fig. 2, we show the contour plots of $\log _{10}\left(\sigma_{\min }([A-z I B])\right)$ and the location of $z^{\star}=1.3843 \pm 0.9738 j$.

In addition to the above two numerical examples, we carried out numerous experiments for randomly generated pairs $A \in \mathbf{R}^{n \times n}$ and $B \in \mathbf{R}^{n \times m}$. If we generate $A$ and $B$ randomly, it can be observed that the number of the global optimizers in (2) is likely to be one or two. More precisely, in the former case the global optimizer is a real number and in the latter case two global optimizers form a complex conjugate pair. In our tested numerical experiments, in every case where the number of the global optimizers is one, the resulting variables $H$ and $\widehat{H}$ is rank-one as we see in the results for "Markov_Chain104." Similarly, in every case where the number of the global optimizers is two, the resulting variables $H$ and $\widehat{H}$ as well as $H_{1}$ and $\widehat{H}_{1}$ are all rank-two as we see in the results for "Grcar104." Namely, in our tested numerical examples, the assumption in Theorem 1 is always satisfied.

To examine the effectiveness of Theorem 1 and Corollary 1 more carefully, we solved the SDP (16) for the matrix pair $A \in \mathbf{R}^{4 \times 4}$ and $B \in \mathbf{R}^{4 \times 1}$ derived in [4]. These matrices are constructed via optimization over the free parameters in the matrix $A$ so that the problem (2) has four global optimizers (see Fig. 3).

By solving (16) for this matrix pair, we obtained $\varepsilon_{2}^{\star}=0.4897$. The computation time was 0.28 [sec]. Again aiming at obtaining exactness certificate of the resulting lower bound, we first examined the rank of the resulting $H$ and $\widehat{H}$. From the singular values of $H$ and $\widehat{H}$, it turned out that we can regard both matrices $H$ and $\widehat{H}$ to be of rank-four. The matrices $H_{1}$ and $\widehat{H}_{1}$ were also confirmed to be full-column rank. Hence we can conclude from Theorem 1 that $\varepsilon_{2}^{\star}=\varepsilon^{\star}$. To obtain the global optimizers via Corollary 1 , we next computed the matrix $X$ in $(23)$, which turned out to be

$$
X=\left[\begin{array}{rrrr}
2.5775 & 3.1441 & 0.0029 & 0.0010 \\
-3.1441 & 2.5775 & -0.0013 & 0.0056 \\
-0.0008 & 0.0028 & 1.0395 & 0.1646 \\
-0.0054 & 0.0034 & 0.1762 & -2.1273
\end{array}\right]
$$

The eigenvalues of this matrix are $2.5775 \pm 3.1441 j,-2.1365$ and 1.0487 . We can confirm that the value $\sigma_{\min }([z I-A B])$ on these eigenvalues are all 0.4897 . Hence, all of these eigenvalues are surely the global optimizers of (2).

We finally examined the computational burden to solve (16) via numerical experiments. We randomly generated $A \in \mathbf{R}^{n \times n}$ and $B \in \mathbf{R}^{n \times m}$ by using MATLAB command rand so that all of their elements lie on $[-1,1]$. For each $(n, m) \in\{(5,3),(10,6),(15,9)$, $(20,12),(25,15),(30,18)\}$, we thus generated ten pairs of $(A, B)$ and examined the average CPU time to solve (16). The results are shown in Fig. 4. It should be noted that, again, the rank condition in Theorem 1 is satisfied in all 60 examples tested here. 
Remark 2 Even though we have restricted our attention to the second-degree polynomial approximation, it is of course possible to take higher-degree polynomial approximation in (14) and derive SDPs corresponding to (15). Moreover, we can derive dual SDPs and associated rank conditions for the exactness verification in a conformable form to Lemma 1 and Theorem 1. In particular, we can prove that those rank conditions become more likely to be satisfied by increasing the degree $N$ as desired. However, since extensive numerical experiments illustrate that the second degree polynomial approximation surely works fine in practice, we omit the detailed discussions for higher-degree polynomial approximation.

\section{Computation of the Similarity Transformation Ma- trix to Maximize the DTUC}

For given controllable pair $A \in \mathbf{C}^{n \times n}$ and $B \in \mathbf{C}^{n \times m}$, it is known that its DTUC correlates to the numerical difficulties of the associated pole placement problem. More precisely, it is shown in [16] that the problem to compute the state-feedback gain that assigns the closed-loop poles to $\mathcal{P}=\left\{\lambda_{1}, \cdots, \lambda_{n}\right\}$ becomes numerically ill-conditioned when the factor $d$ is large, where

$$
d(A, B):=\frac{1}{\min _{i} \sigma_{\min }\left(\left[A-\lambda_{i} I B\right]\right)} .
$$

From this form, it is obvious that the pole placement problem for $(A, B)$ is likely to be ill-conditioned if its DTUC is small $[13,16]$.

As an attempt to achieve conditioning of the pole placement problem for the pair $(A, B)$, let us consider the problem to compute the similarity transformation matrix $T$ that maximizes the lower bound of the DTUC of the pair $(\widehat{A}, \widehat{B})=\left(T^{-1} A T, T^{-1} B\right)$. As clarified later on, the optimal $T$ in this context can be determined by solving a generalized eigenvalue problem (GEP).

To see this, let us slightly modify the SDP (15) and define $\widehat{\varepsilon}_{2}^{\star}$ as follows:

$$
\widehat{\varepsilon}_{2}^{\star}:=\max _{X_{0}, X_{1}, X_{2}, G} \varepsilon \quad \text { subject to (15) with } D=0 \text {. }
$$

Roughly speaking, letting $D=0$ implies that we seek for the optimum of (3) over the whole complex plane. In our preceding work [7], it is illustrated through extensive numerical experiments that the SDP (26) still performs well and $\widehat{\varepsilon}_{2}^{\star}$ is very close to the exact value $\varepsilon^{\star}$.

With this in mind, let us consider the problem to compute the similarity transformation matrix $T$ that maximizes the corresponding quantity $\widehat{\varepsilon}_{2}^{\star}$ of $(\widehat{A}, \widehat{B})=\left(T^{-1} A T, T^{-1} B\right)$. This problem can be stated as follows:

$$
\begin{aligned}
& \widehat{\varepsilon}_{2}^{\star}=\max _{X_{0}, X_{1}, X_{2}, G, T} \varepsilon \quad \text { subject to } \\
& \begin{array}{l}
{\left[\begin{array}{cc:cc}
-\varepsilon^{2} I-X_{0} & 0 & -\frac{1}{2} X_{1} & -T^{*} A^{*} T^{*-1} \\
0 & T^{-1}\left(A T T^{*} A^{*}+B B^{*}\right) T^{*-1}+X_{0} & 0 & \frac{1}{2} X_{1} \\
\hdashline-\frac{1}{2} X_{1} & 0 & I-X_{2} & 0 \\
-T^{-1} A T & \frac{1}{2} X_{1} & 0 & X_{2}
\end{array}\right]+\left[\begin{array}{cc}
0 & G \\
G^{*} & 0
\end{array}\right] \geq 0,} \\
X_{0}, X_{1}, X_{2} \in \mathbf{H}_{n}, \quad G \in \mathbf{S}_{2 n} .
\end{array}
\end{aligned}
$$


By applying congruence transformation with $\operatorname{diag}(T, T, T, T)$ to $(27)$, we have

$$
\begin{aligned}
& \widehat{\varepsilon}_{2}^{\star}=\max _{\widehat{X}_{0}, \widehat{X}_{1}, \widehat{X}_{2}, \widehat{G}, V} \varepsilon \quad \text { subject to } \\
& \begin{array}{l}
{\left[\begin{array}{cc:cc}
-\varepsilon^{2} V-\widehat{X}_{0} & 0 & -\frac{1}{2} \widehat{X}_{1} & -V A^{*} \\
0 & A V A^{*}+B B^{*}+\widehat{X}_{0} & 0 & \frac{1}{2} \widehat{X}_{1} \\
\hdashline-\frac{1}{2} \widehat{X}_{1} & 0 & V-\widehat{X}_{2} & 0 \\
-A V & \frac{1}{2} \widehat{X}_{1} & 0 & \widehat{X}_{2}
\end{array}\right]+\left[\begin{array}{cc}
0 & \widehat{G} \\
\widehat{G}^{*} & 0
\end{array}\right] \geq 0,} \\
\widehat{X}_{0}, \widehat{X}_{1}, \widehat{X}_{2} \in \mathbf{H}_{n}, \quad \widehat{G} \in \mathbf{S}_{2 n}, \quad V>0 .
\end{array}
\end{aligned}
$$

When deriving (28) from (27), we define $V:=T T^{*}>0, \widehat{X}_{i}=T X_{i} T^{*}(i=0,1,2)$ and $\widehat{G}=\operatorname{diag}(T, T) G \operatorname{diag}(T, T)^{*}$. It can be seen that the matrix inequality (28) is an LMI with respect to the matrix variables. It follows that the matrix $T$ that maximizes $\widehat{\varepsilon}_{2}^{\star}$ for the pair $(\widehat{A}, \widehat{B})=\left(T^{-1} A T, T^{-1} B\right)$ can be determined by solving the GEP (28). Once we have solved (28), the optimal $T$ can be determined from $V>0$ via $T T^{*}=V$.

Even though the maximization is performed with respect to the lower bound of the DTUC, we can obtain a desired similarity transformation matrix $T$ efficiently by solving the GEP (28). Similar results would not follow if we pursue the direction of the linear algebra algorithms $[4,9,10]$. This illustrates in part the usefulness of the proposed LMI-based approach over the existing approaches $[4,9,10]$.

Remark 3 From the structure of the LMI (28), we see that if (28) holds with $\widehat{X}_{i}=$ $\widetilde{X}_{i}(i=0,1,2), \widehat{G}=\widetilde{G}, V=\widetilde{V}$ and $\varepsilon=\widetilde{\varepsilon}$, then this LMI holds for the same value $\varepsilon=\widetilde{\varepsilon}$ by letting $\widehat{X}_{i}=\alpha^{2} \widetilde{X}_{i},(i=0,1,2), \widehat{G}=\alpha^{2} \widetilde{G}, V=\alpha^{2} \widetilde{V}$ with $0<\alpha \leq 1$. This feature stems from the definition of the DTUC (2), which implies that the DTUC of $\left(Z^{-1} A Z, Z^{-1} B\right)$ is not less than that of $(\widehat{A}, \widehat{B})=\left(T^{-1} A T, T^{-1} B\right)$ if we simply let $Z=$ $\alpha T(0<\alpha \leq 1)$. This clearly indicates that, when we consider the problem to compute the similarity transformation matrix, it is necessary to bound the norm of $T^{*-1} T^{-1}\left(=V^{-1}\right)$ for the problem to be well-posed. In view of these facts, we impose an additional constraint $V \geq \beta I(0<\beta \leq 1)$ when we solve the GEP (28). This works effectively also to avoid numerical instability of the GEP (28).

Example 2 To illustrate the usefulness of the suggested computation method of the similarity transformation matrix, let us consider the following matrices $A$ and $B$ :

$$
A=\left[\begin{array}{rrrrr}
1.3504 & 1.4918 & 0 & 0 & 0 \\
0.6703 & -0.8066 & 1.4918 & 0 & 0 \\
0 & 0.6703 & 0.3205 & 1.4918 & 0 \\
0 & 0 & 0.6703 & -0.0421 & 1.4918 \\
0 & 0 & 0 & 0.6703 & 1.1739
\end{array}\right], \quad B=\left[\begin{array}{rr}
-1.4986 & -0.3308 \\
-0.0503 & 0.7952 \\
0.5530 & -0.7848 \\
0.0835 & -1.2631 \\
1.5775 & 0.6667
\end{array}\right]
$$

This matrix data can be found at [27] with the name "Hatano52". For this matrix pair, we first compute the lower bound $\varepsilon_{2}^{\star}$ of the DTUC by solving the SDP (16), which turns out to be $\varepsilon_{2}^{\star}=0.3958$. In this case the resulting optimal variables $H$ and $\widehat{H}$ are both rank-one, and hence from Corollary 2 we can conclude that the exact DTUC is 0.3958 . 
The optimizer $z^{\star}$ of (2) can also be computed as $z^{\star}=2.0934$. In Figs 5 and 6 , we show contour plots of $\log _{10}\left(\sigma_{\min }([A-z I B])\right)$.

To increase the DTUC via similarity transformation, we next solved the GEP (28) with an additional constraint

$$
V=T T^{*} \geq 10^{-6} I \text {. }
$$

The resulting lower bound is $\widehat{\varepsilon}_{2}^{\star}=10.0065$ and the transformation matrix is

$$
T=\left[\begin{array}{rrrrr}
0.0724 & -0.0027 & -0.0217 & 0.0013 & -0.0763 \\
-0.0027 & 0.0348 & -0.0251 & -0.0402 & 0.0102 \\
-0.0217 & -0.0251 & 0.0483 & 0.0408 & 0.0097 \\
0.0013 & -0.0402 & 0.0408 & 0.0724 & -0.0175 \\
-0.0763 & 0.0102 & 0.0097 & -0.0175 & 0.0878
\end{array}\right] .
$$

In particular, by means of the SDP (16), Theorem 1 and Corollary 1 , we confirmed that the DTUC of $(\widehat{A}, \widehat{B})=\left(T^{-1} A T, T^{-1} B\right)$ is surely 10.0065. The global optimizers turn out to be $5.1740 \pm 6.3107 j$. In Figs 7 and 8, we show contour plots of $\log _{10}\left(\sigma_{\min }([\widehat{A}-z I \widehat{B}])\right)$. In stark contrast with the behavior of $\sigma_{\min }([A-z I B])$ around the optimizer $z^{\star}=2.0934$, we see from Figs 7 and 8 that the behavior of $\sigma_{\min }([\widehat{A}-z I \widehat{B}])$ becomes considerably flat around the optimizer $z^{\star}=5.1740 \pm 6.3107 j$.

To confirm the effectiveness of the suggested computation method more convincingly, we next consider very simple similarity transformations. First, we take $T=T_{\mathrm{D}}$, where $T_{\mathrm{D}}$ is the matrix that transforms $A$ to be diagonal ${ }^{2}$. The DTUC of the transformed pair is 0.2858 and smaller than the original one 0.3958. Second, we take $T=\alpha I$ for comparison. As we stated in Remark 3, the DTUC of $(\widehat{A}, \widehat{B})=\left(A, \alpha^{-1} B\right)$ is monotonically nonincreasing with respect to $\alpha>0$. Thus the matrix $T=10^{-3} I$ is tested here in view of the constraint (30). It turns out that the resulting DTUC of $(\widehat{A}, \widehat{B})=\left(A, 10^{3} B\right)$ is 0.4817 , which is very small in comparison with 10.0065 achieved by $T$ in (31).

We finally compute the factor $d$ given in (25) for the set of closed-loop poles $\mathcal{P}:=$ $\{-1,-2,-3,-4,-5\}$ as a trial. It follows that $d(A, B)=2.1733, d\left(A, 10^{3} B\right)=1.0586$ and $d\left(T^{-1} A T, T^{-1} B\right)$ with $T$ given in (31) is 0.0995. Namely, the factor $d$ has been reduced significantly. This illustrates the usefulness of the suggested computation method of the similarity transformation matrices by means of systematic convex optimization.

\section{Conclusion}

In this paper, we considered the problem to compute the distance to uncontrollability (DTUC) of a given controllable pair $(A, B)$ by means of LMIs. We first reduced this problem into a robust SDP and then derived a numerically tractable SDP for a lower bound computation. We next showed that this lower bound can be characterized by a very concise dual SDP. Based on this dual SDP, we further derived a viable test to verify that the computed lower bound coincides with the exact DTUC. In the latter part of the paper, on the other hand, we showed that the similarity transformation matrix that maximizes a lower bound of the DTUC of the transformed pair can be computed

\footnotetext{
${ }^{2}$ The matrix $T_{\mathrm{D}}$ satisfies $\left\|T_{\mathrm{D}}\right\|\left\|T_{\mathrm{D}}^{-1}\right\|=4.9010$. Note that similarity transformation to maximize DTUC is typically needed in the case where $A$ is far from normal, and hence $\left\|T_{\mathrm{D}}\right\|\left\|T_{\mathrm{D}}^{-1}\right\|$ is large.
} 
by solving a generalized eigenvalue problem. It is expected that this computation of the similarity transformation matrix leads to an effective and efficient conditioning of the pole placement problem for the pair $(A, B)$.

\section{Acknowledgement}

The author is grateful to D. Henrion for his helpful comments. He also would like to thank anonymous reviewers for their careful reading and valuable suggestions.

\section{Appendix}

Proof of Lemma 1: The result basically follows from convex duality theory [1, 24]. To be more precise, we first note that the SDP (15) can be rewritten equivalently as follows:

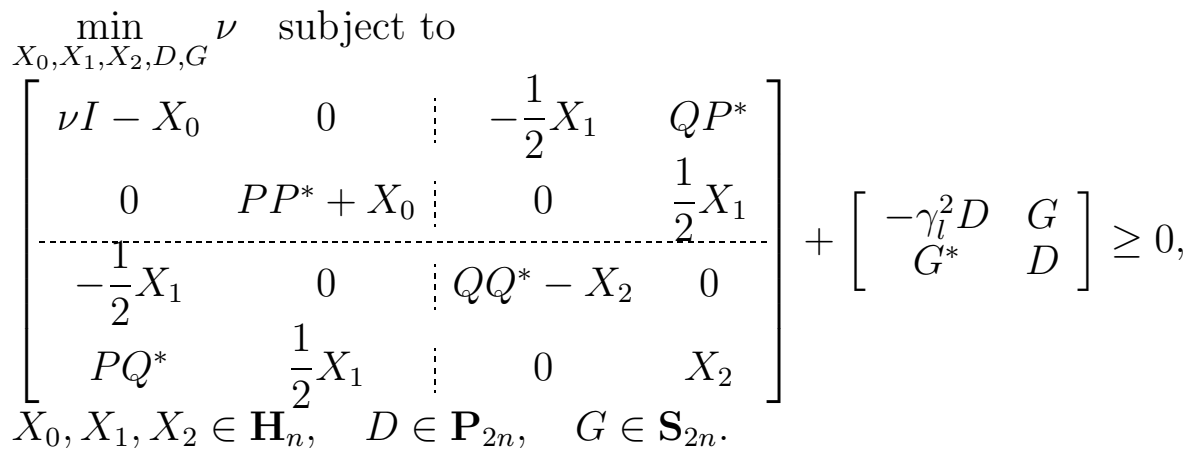

If we denote the optimal value of this SDP by $\nu^{\star}(\leq 0)$, it is apparent that $\varepsilon_{2}^{\star}=\sqrt{-\nu^{\star}}$. In addition, if we denote the left-hand side of (32) by $\mathcal{M}\left(\nu, X_{0}, X_{1}, X_{2}, D, G\right)$, we see that there exist $X_{0}, X_{1}, X_{2} \in \mathbf{H}_{n}, D>0$ and $G \in \mathbf{S}_{2 n}$ such that $\mathcal{M}\left(\nu, X_{0}, X_{1}, X_{2}, D, G\right)>0$, i.e., the SDP (32) satisfies the Slater's constraint qualification [24]. For example, by letting

$$
\nu=4 \gamma_{l}^{2}, \quad X_{0}=2 \gamma_{l}^{2} I_{n}, \quad X_{1}=0, \quad X_{2}=0, \quad D=I_{2 n}, \quad G=\left[\begin{array}{cc}
0 & -Q P^{*} \\
P Q^{*} & 0
\end{array}\right],
$$

we can confirm that the constraint qualification is surely satisfied for any $P$ and $Q$.

With this in mind, let us introduce the Lagrange multipliers $\mathcal{H} \in \mathbf{P}_{4 n}$ and $\mathcal{J} \in \mathbf{P}_{2 n}$ and define the Lagrangian $\mathcal{L}$ as follows:

$$
\mathcal{L}\left(\nu, X_{0}, X_{1}, X_{2}, D, G, \mathcal{H}, \mathcal{J}\right):=\nu-\operatorname{trace}\left(\mathcal{M}\left(\nu, X_{0}, X_{1}, X_{2}, D, G\right) \mathcal{H}\right)-\operatorname{trace}(D \mathcal{J}) .
$$

By partitioning $\mathcal{H}$ as

$$
\mathcal{H}:=\left[\begin{array}{cccc}
\mathcal{H}_{11} & \mathcal{H}_{12} & \mathcal{H}_{13} & \mathcal{H}_{14} \\
\mathcal{H}_{12}^{*} & \mathcal{H}_{22} & \mathcal{H}_{23} & \mathcal{H}_{24} \\
\mathcal{H}_{13}^{*} & \mathcal{H}_{23}^{*} & \mathcal{H}_{33} & \mathcal{H}_{34} \\
\mathcal{H}_{14}^{*} & \mathcal{H}_{24}^{*} & \mathcal{H}_{34}^{*} & \mathcal{H}_{44}
\end{array}\right], \mathcal{H}_{i i} \in \mathbf{S}_{n}(i=1, \cdots, 4),
$$


we see that the Lagrangian can be rewritten, equivalently, as follows:

$$
\begin{aligned}
& \mathcal{L}\left(\nu, X_{0}, X_{1}, X_{2}, D, G, \mathcal{H}, \mathcal{J}\right) \\
& =\nu\left(1-\operatorname{trace}\left(\mathcal{H}_{11}\right)\right)+\operatorname{trace}\left(X_{0}\left(\mathcal{H}_{11}-\mathcal{H}_{22}\right)\right) \\
& \quad+\frac{1}{2} \operatorname{trace}\left(X_{1}\left(\mathcal{H}_{13}+\mathcal{H}_{13}^{*}-\mathcal{H}_{24}-\mathcal{H}_{24}^{*}\right)\right)+\operatorname{trace}\left(X_{2}\left(\mathcal{H}_{33}-\mathcal{H}_{44}\right)\right) \\
& +\operatorname{trace}\left(D\left(\gamma_{l}^{2}\left[\begin{array}{ll}
\mathcal{H}_{11} & \mathcal{H}_{12} \\
\mathcal{H}_{12}^{*} & \mathcal{H}_{22}
\end{array}\right]-\left[\begin{array}{cc}
\mathcal{H}_{33} & \mathcal{H}_{34} \\
\mathcal{H}_{34}^{*} & \mathcal{H}_{44}
\end{array}\right]-\mathcal{J}\right)\right) \\
& +\operatorname{trace}\left(G\left(\left[\begin{array}{cc}
\mathcal{H}_{13} & \mathcal{H}_{14} \\
\mathcal{H}_{23} & \mathcal{H}_{24}
\end{array}\right]-\left[\begin{array}{cc}
\mathcal{H}_{13}^{*} & \mathcal{H}_{23}^{*} \\
\mathcal{H}_{14}^{*} & \mathcal{H}_{24}^{*}
\end{array}\right]\right)\right) \\
& \quad-\operatorname{trace}\left(\left[\begin{array}{ll}
P P^{*} & P Q^{*} \\
Q P^{*} & Q Q^{*}
\end{array}\right]\left[\begin{array}{ll}
\mathcal{H}_{22} & \mathcal{H}_{14}^{*} \\
\mathcal{H}_{14} & \mathcal{H}_{33}
\end{array}\right]\right) .
\end{aligned}
$$

Hence, for this Lagrangian to be bounded below for any $\nu \in \mathbf{R}, X_{0}, X_{1}, X_{2} \in \mathbf{H}_{n}$, $D \in \mathbf{H}_{2 n}$ and $G \in \mathbf{S}_{2 n}$, the Lagrange multipliers should satisfy

$$
\begin{aligned}
& \operatorname{trace}\left(\mathcal{H}_{11}\right)=1, \quad \mathcal{H}_{11}=\mathcal{H}_{22}, \quad \mathcal{H}_{13}+\mathcal{H}_{13}^{*}=\mathcal{H}_{24}+\mathcal{H}_{24}^{*}, \quad \mathcal{H}_{33}=\mathcal{H}_{44}, \\
& \gamma_{l}^{2}\left[\begin{array}{ll}
\mathcal{H}_{11} & \mathcal{H}_{12} \\
\mathcal{H}_{12}^{*} & \mathcal{H}_{22}
\end{array}\right]-\left[\begin{array}{ll}
\mathcal{H}_{33} & \mathcal{H}_{34} \\
\mathcal{H}_{34}^{*} & \mathcal{H}_{44}
\end{array}\right]-\mathcal{J}=0, \quad\left[\begin{array}{lll}
\mathcal{H}_{13} & \mathcal{H}_{14} \\
\mathcal{H}_{23} & \mathcal{H}_{24}
\end{array}\right]=\left[\begin{array}{ccc}
\mathcal{H}_{13}^{*} & \mathcal{H}_{23}^{*} \\
\mathcal{H}_{14}^{*} & \mathcal{H}_{24}^{*}
\end{array}\right] .
\end{aligned}
$$

In addition, since the Slater's constraint qualification is satisfied in (32), we can conclude that $\nu^{*}$ can be characterized via dual SDP given as follows:

$$
\nu^{\star}=\max _{\mathcal{H} \geq 0, \mathcal{J} \geq 0}-\operatorname{trace}\left(\left[\begin{array}{cc}
P P^{*} & P Q^{*} \\
Q P^{*} & Q Q^{*}
\end{array}\right]\left[\begin{array}{ll}
\mathcal{H}_{22} & \mathcal{H}_{14}^{*} \\
\mathcal{H}_{14} & \mathcal{H}_{33}
\end{array}\right]\right) \text { subject to (33). }
$$

Moreover, by rearranging (33) and considering the relation $\varepsilon_{2}^{\star}=\sqrt{-\nu^{\star}}$, we have

$$
\varepsilon_{2}^{\star 2}=\min _{\mathcal{H}} \operatorname{trace}\left(\left[\begin{array}{ll}
P P^{*} & P Q^{*} \\
Q P^{*} & Q Q^{*}
\end{array}\right]\left[\begin{array}{ll}
\mathcal{H}_{11} & \mathcal{H}_{14}^{*} \\
\mathcal{H}_{14} & \mathcal{H}_{33}
\end{array}\right]\right)
$$

subject to

$$
\mathcal{H}=\left[\begin{array}{llll}
\mathcal{H}_{11} & \mathcal{H}_{12} & \mathcal{H}_{13} & \mathcal{H}_{14} \\
\mathcal{H}_{12}^{*} & \mathcal{H}_{11} & \mathcal{H}_{14}^{*} & \mathcal{H}_{13} \\
\mathcal{H}_{13} & \mathcal{H}_{14} & \mathcal{H}_{33} & \mathcal{H}_{34} \\
\mathcal{H}_{14}^{*} & \mathcal{H}_{13} & \mathcal{H}_{34}^{*} & \mathcal{H}_{33}
\end{array}\right] \geq 0, \gamma_{l}^{2}\left[\begin{array}{ll}
\mathcal{H}_{11} & \mathcal{H}_{12} \\
\mathcal{H}_{12}^{*} & \mathcal{H}_{11}
\end{array}\right] \geq\left[\begin{array}{ll}
\mathcal{H}_{33} & \mathcal{H}_{34} \\
\mathcal{H}_{34}^{*} & \mathcal{H}_{33}
\end{array}\right], \operatorname{trace}\left(\mathcal{H}_{11}\right)=1
$$

To complete the proof, it remains to show that the SDP (34) can be rewritten equivalently as (16). To this end, we note that $\mathcal{H} \geq 0$ in (34b) is equivalent to

$$
T_{\mathcal{H}} \mathcal{H} T_{\mathcal{H}}^{T}=\left[\begin{array}{llll}
\mathcal{H}_{11} & \mathcal{H}_{14} & \mathcal{H}_{12} & \mathcal{H}_{13} \\
\mathcal{H}_{14}^{*} & \mathcal{H}_{33} & \mathcal{H}_{13} & \mathcal{H}_{34}^{*} \\
\mathcal{H}_{12}^{*} & \mathcal{H}_{13} & \mathcal{H}_{11} & \mathcal{H}_{14}^{*} \\
\mathcal{H}_{13} & \mathcal{H}_{34} & \mathcal{H}_{14} & \mathcal{H}_{33}
\end{array}\right] \geq 0, \quad T_{\mathcal{H}}:=\left[\begin{array}{cccc}
I & 0 & 0 & 0 \\
0 & 0 & 0 & I \\
0 & I & 0 & 0 \\
0 & 0 & I & 0
\end{array}\right]
$$

Since $\mathcal{H}_{12}, \mathcal{H}_{13}$ and $\mathcal{H}_{34}$ are irrelevant to (34a), and since these variables enter into the off-diagonal blocks of (35) and the second one in (34b), we see that they can be taken as zeros without loss of generality. This observation leads us to the desired dual SDP (16), where the variables $H_{11}, H_{12}$ and $H_{22}$ correspond to $\mathcal{H}_{11}, \mathcal{H}_{14}^{*}$ and $\mathcal{H}_{33}$, respectively. 


\section{References}

[1] V. Balakrishnan and L. Vandenberghe, "Semidefinite Programming Duality and Linear Time-Invariant Systems," IEEE Trans. Automatic Control, Vol. 48, No. 1, pp. 30-41 (2003).

[2] P. A. Bliman, "A Convex Approach to Robust Stability for Linear Systems with Uncertain Scalar Parameters", SIAM J. Control and Optimization, Vol. 42, No. 6, pp. 2016-2042 (2004).

[3] S. P. Boyd, L. E. Ghaoui, E. Feron, and V. Balakrishnan, Linear Matrix Inequalities in System and Control Theory, SIAM (1994).

[4] J. V. Burke, A. S. Lewis and M. L. Overton, "Pseudospectral Components and the Distance to Uncontrollability," SIAM Journal on Matrix Analysis and Applications, Vol. 26, No. 2, pp. 350-361 (2004).

[5] G. Chen and T. Sugie, "New Upper Bound of the Real $\mu$ Based on the Parameter Dependent Multiplier," Proc. Conference on Decision and Control, pp. 1293-1294 (1996).

[6] Y. Ebihara, K. Maeda and T. Hagiwara, "Generalized $\mathcal{S}$-procedure for Inequality Conditions on One-Vector-Lossless Sets and Linear System Analysis," Proc. Conference on Decision and Control, pp. 1272-1277 (2004).

[7] Y. Ebihara and T. Hagiwara, "Computing the Distance to Uncontrollability via LMIs: Lower and Upper Bounds Computation and Exactness Verification," Proc. Conference on Decision and Control, pp. 5772-5777 (2006).

[8] R. Eising, "Between Controllable and Uncontrollable," Systems and Control Letters, Vol. 4, No. 5, pp. 263-264 (1984).

[9] M. Gu, "New Methods for Estimating the Distance to Uncontrollability," SIAM Journal on Matrix Analysis and Applications, Vol. 21, No. 3, pp. 989-1003 (2000).

[10] M. Gu, E. Mengi, M. L. Overton, J. Xia, and J. Zhu, "Fast Methods for Estimating the Distance to Uncontrollability," SIAM Journal on Matrix Analysis and Applications, Vol. 28, No. 2, pp. 477-502 (2006).

[11] D. Henrion and G. Meinsma, "Rank-One LMIs and Lyapunov's Inequality," IEEE Trans. Automatic Control, Vol. 46, No. 8, pp. 1285-1288 (2001).

[12] D. Henrion, M. Šebeek and V. Kučera, "Positive Polynomials and Robust Stabilization with Fixed-Order Controllers," IEEE Trans. Automatic Control, Vol. 48, No. 7, pp. 1178-1186 (2003).

[13] N. J. Higham, M. Konstantinov, V. Mehrmann and P. Petkov, "The Sensitivity of Computational Control Problems," IEEE Control System Magazine, Vol. 24, No. 1, pp. 28-43 (2004). 
[14] T. Iwasaki, G. Meinsma and M. Fu, "Generalized $\mathcal{S}$-procedure and Finite Frequency KYP Lemma," Mathematical Problems in Engineering, Vol. 6, pp. 305-320 (2000).

[15] T. Iwasaki and S. Hara, "Generalized KYP Lemma: Unified Frequency Domain Inequalities with Design Applications," IEEE Trans. Automatic Control, Vol. 50, No. 1, pp. 41-59 (2005).

[16] V. Mehrmann and H. Xu, "An Analysis of the Pole Placement Problem II: The Multi-Input Case," Electronic Trans. Numerical Analysis, Vol. 5, pp. 77-97 (1997).

[17] A. Ohara and Y. Sasaki, "On Solvability and Numerical Solutions of ParameterDependent Differential Matrix Inequality," Proc. Conference on Decision and Control, pp. 3593-3594 (2001).

[18] A. Packard and J. Doyle, "The Complex Structured Singular Value," Automatica, Vol. 29, pp. 71-109 (1993).

[19] C. C. Paige, "Properties of Numerical Algorithms Related to Computing Controllability," IEEE Trans. Automatic Control, Vol. 26, No. 1, pp. 130-138 (1981).

[20] A. Rantzer, "On the Kalman-Yakubovich-Popov Lemma", Systems and Control Letters, Vol. 28, pp. 7-10 (1996).

[21] C. W. Scherer, "When Are Multiplier Relaxations Exact?" Proc. the 4th IFAC Symposium on Robust Control Design (2003).

[22] C. W. Scherer, "Higher-Order Relaxations for Robust LMI Problems with Verifications for Exactness," Proc. Conference on Decision and Control, pp. 4652-4657 (2003).

[23] C. W. Scherer, "Relaxations for Robust Linear Matrix Inequality Problems with Verifications for Exactness," SIAM Journal on Matrix Analysis and Applications, Vol. 27, No. 2, pp. 365-395 (2005).

[24] C. W. Scherer, "LMI Relaxations in Robust Control," European Journal of Control, Vol. 12, No. 1, pp. 3-29 (2006).

[25] J. F. Sturm: "Using SeDuMi 1.02, a MATLAB Toolbox for Optimization over Symmetric Cones", Optimization Methods and Software, Vol. 11-12, pp. 625-653 (1999).

[26] K. Zhou and J. C. Doyle, Essentials of Robust Control, Prentice Hall (1998).

[27] http://www.cs.nyu.edu/mengi/robust_stability/dist_uncont.html 


\section{List of Figures}

- Figure 1: Contour plots of $\log _{10}\left(\sigma_{\min }([A-z I B])\right)$ for the pair "Markov_Chain104". The circle is of radius $\gamma_{l}=1.5458$. The mark $*$ denotes the location of $z^{\star}=-0.2526$.

- Figure 2: Contour plots of $\log _{10}\left(\sigma_{\min }([A-z I B])\right)$ for the pair "Grcar104". The circle is of radius $\gamma_{l}=4.8412$. The mark $*$ denotes the location of $z^{\star}=1.3842 \pm$ $0.9738 j$.

- Figure 3: Contour plots of $\log _{10}\left(\sigma_{\min }([A-z I B])\right)$ for the pair found at [3]. The circle is of radius $\gamma_{l}=7.9019$. The mark $*$ denotes the location of the global optimizers $z^{\star}=2.5775 \pm 3.1441 j,-2.1365,1.0487$.

- Figure 4: Average CPU time to compute $\varepsilon_{2}^{\star}$ in logarithmic scale.

- Figure 5: Contour plots of $\log _{10}\left(\sigma_{\min }([A-z I B])\right)$ for the pair (29). The circle is of radius $\gamma_{l}=3.8390$. The mark $*$ denote the location of the optimizer $z^{\star}=2.0934$.

- Figure 6: Contour plots of $\log _{10}\left(\sigma_{\min }([A-z I B])\right)$ for the pair (29) in 3D form.

- Figure 7: Contour plots of $\log _{10}\left(\sigma_{\min }([\widehat{A}-z I \widehat{B}])\right)$. The mark $*$ denote the location of the optimizer $z^{\star}=5.1740 \pm 6.3107 j$.

- Figure 8: Contour plots of $\log _{10}\left(\sigma_{\min }([\widehat{A}-z I \widehat{B}])\right)$ in 3D form. 


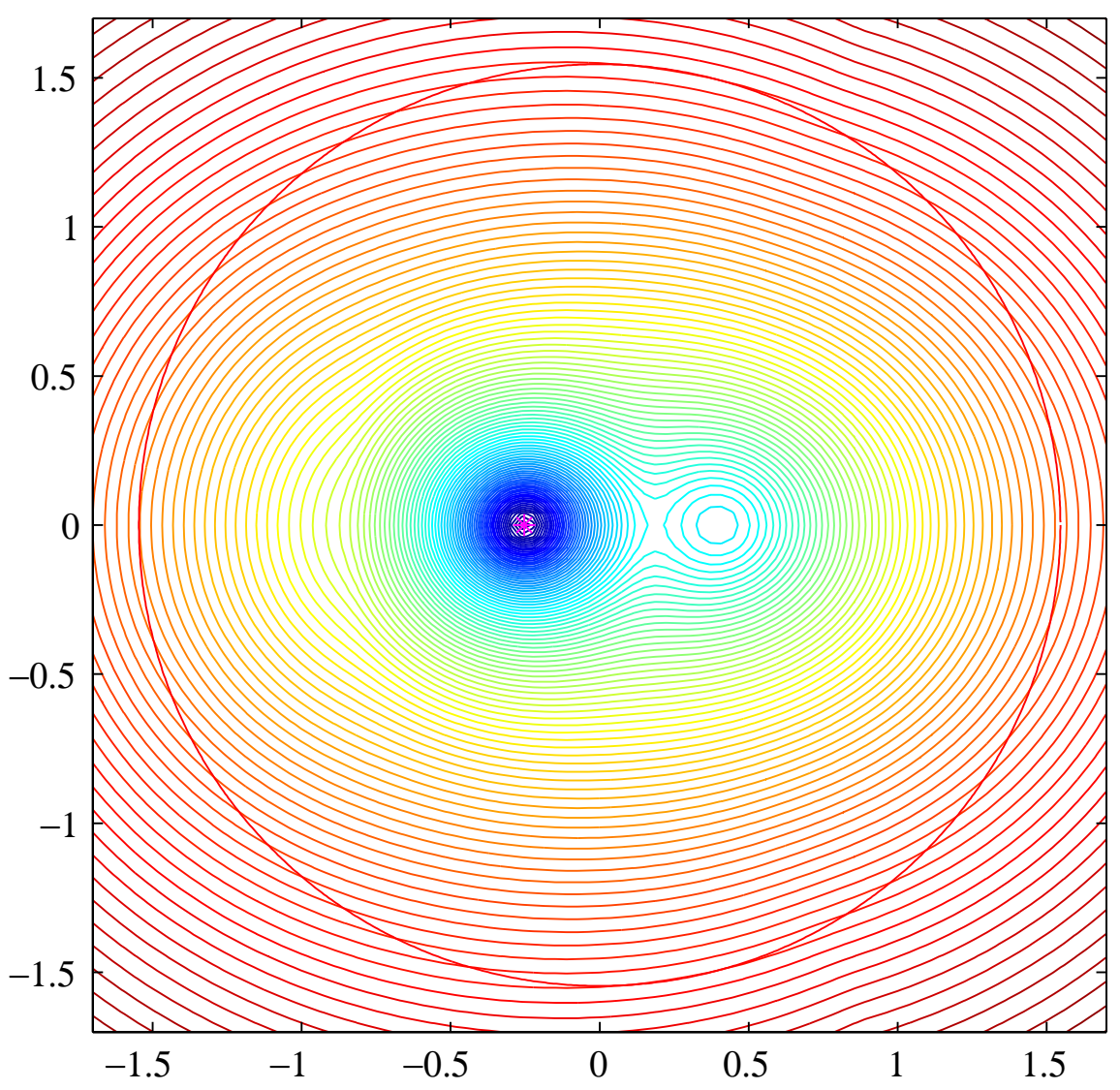

Figure 1: Contour plots of $\log _{10}\left(\sigma_{\min }([A-z I B])\right)$ for the pair "Markov_Chain104". The circle is of radius $\gamma_{l}=1.5458$. The mark $*$ denotes the location of $z^{\star}=-0.2526$. 


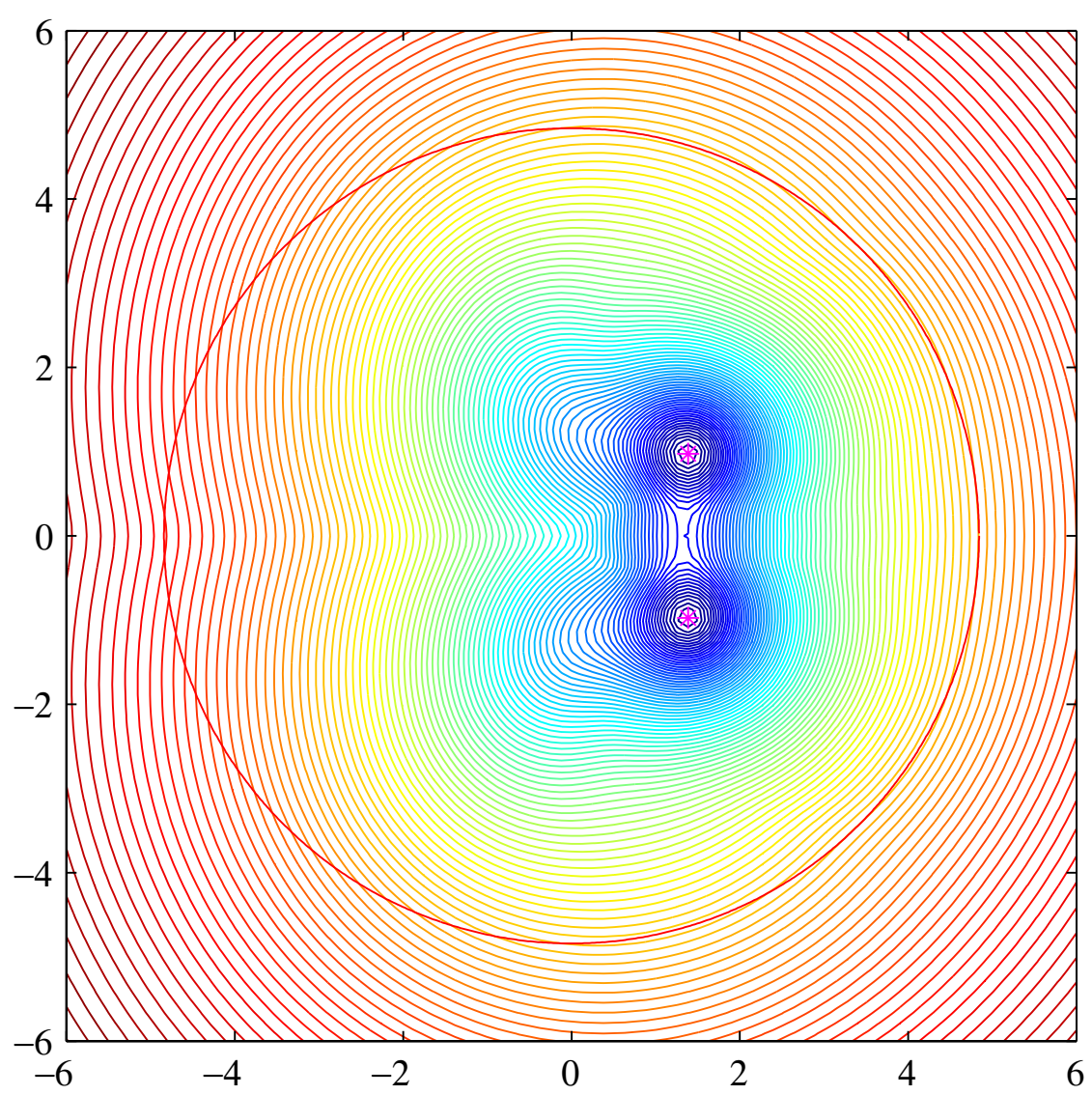

Figure 2: Contour plots of $\log _{10}\left(\sigma_{\min }([A-z I B])\right)$ for the pair "Grcar104". The circle is of radius $\gamma_{l}=4.8412$. The mark $*$ denotes the location of $z^{\star}=1.3842 \pm 0.9738 j$. 


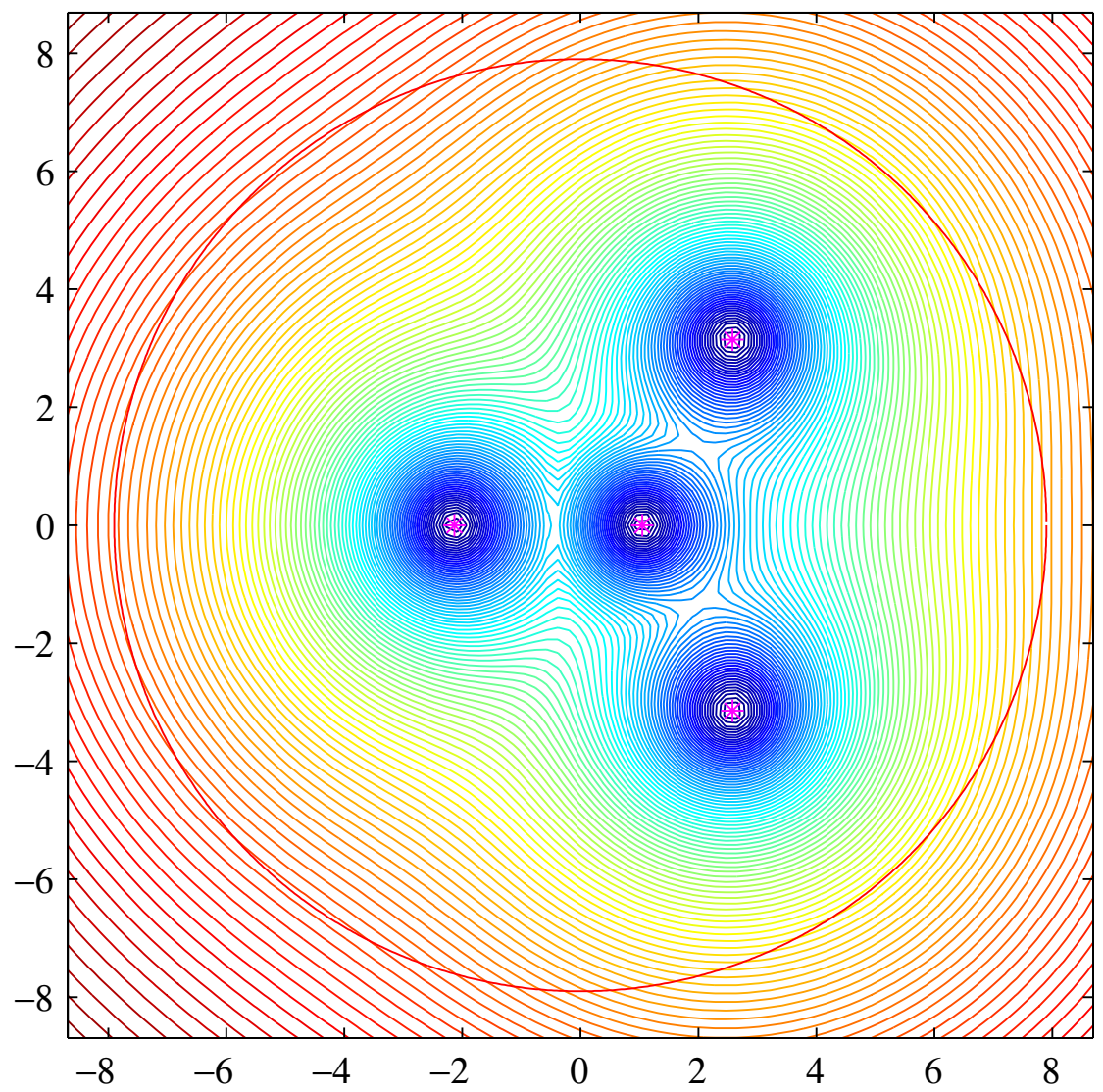

Figure 3: Contour plots of $\log _{10}\left(\sigma_{\min }\left(\left[\begin{array}{lll}A-z I & B\end{array}\right]\right)\right)$ for the pair found at [3]. The circle is of radius $\gamma_{l}=7.9019$. The mark $*$ denotes the location of the global optimizers $z^{\star}=2.5775 \pm 3.1441 j,-2.1365,1.0487$. 


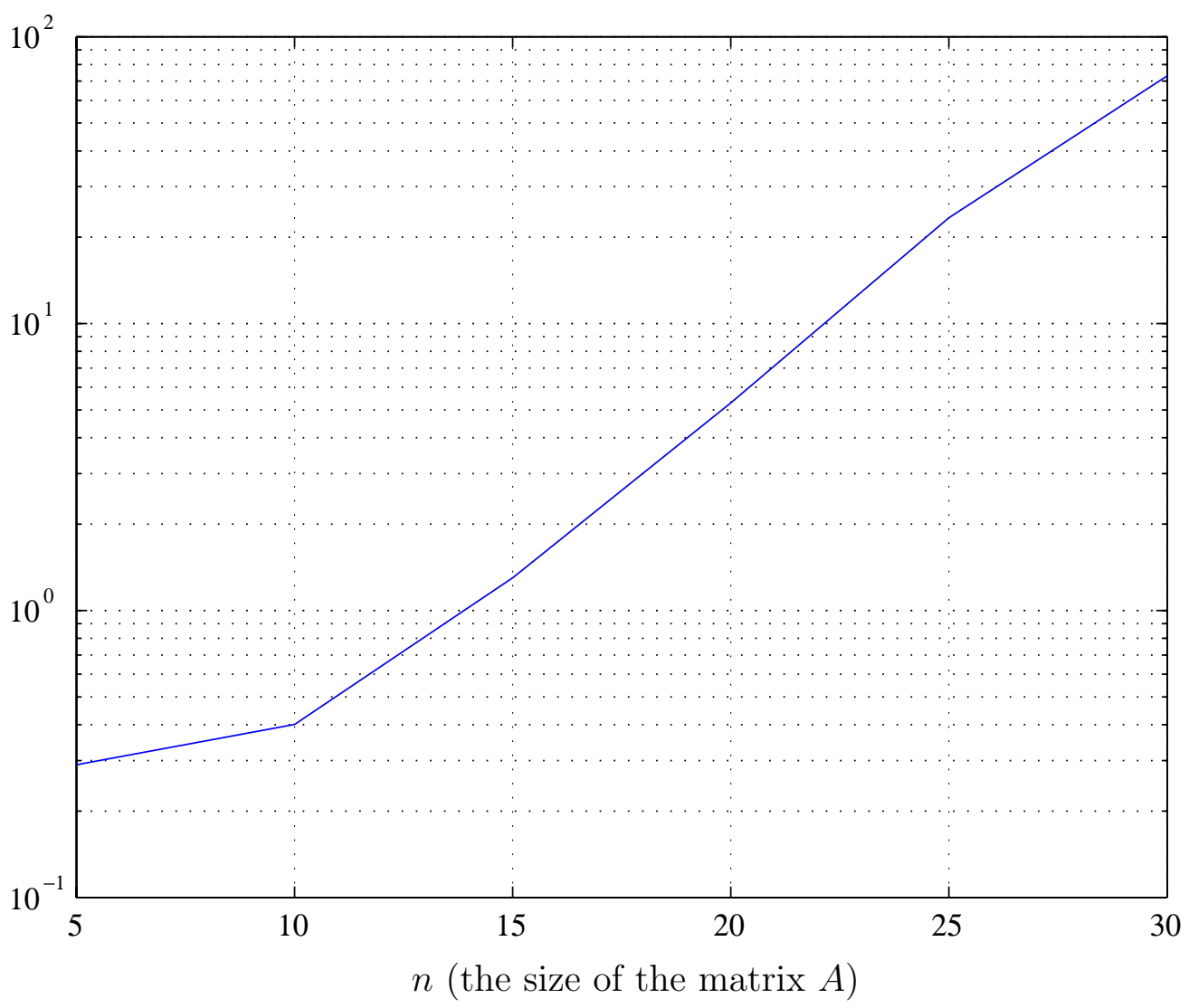

Figure 4: Average CPU time to compute $\varepsilon_{2}^{\star}$ in logarithmic scale. 


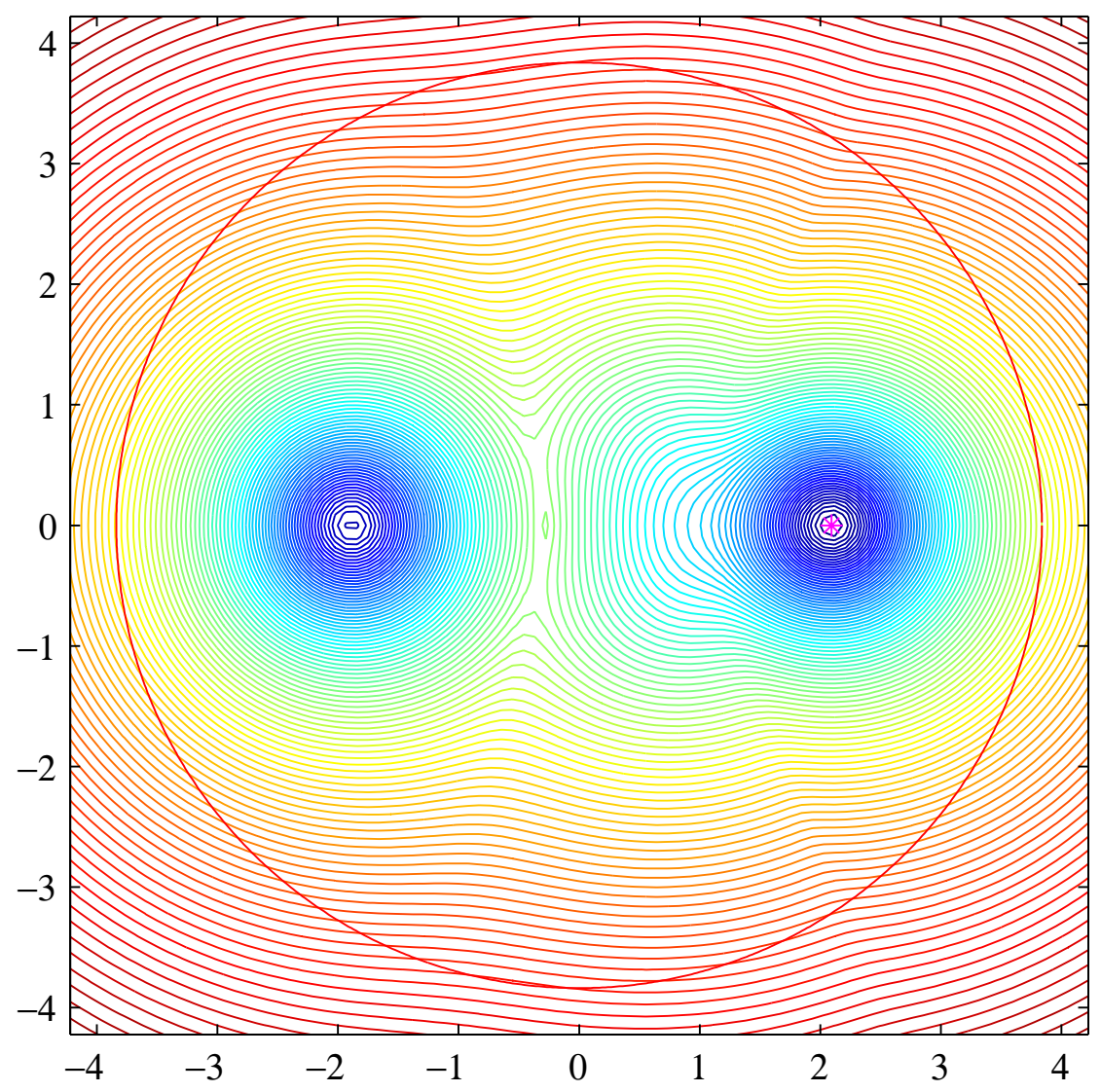

Figure 5: Contour plots of $\log _{10}\left(\sigma_{\min }([A-z I B])\right)$ for the pair (31). The circle is of radius $\gamma_{l}=3.8390$. The mark $*$ denote the location of the optimizer $z^{\star}=2.0934$. 


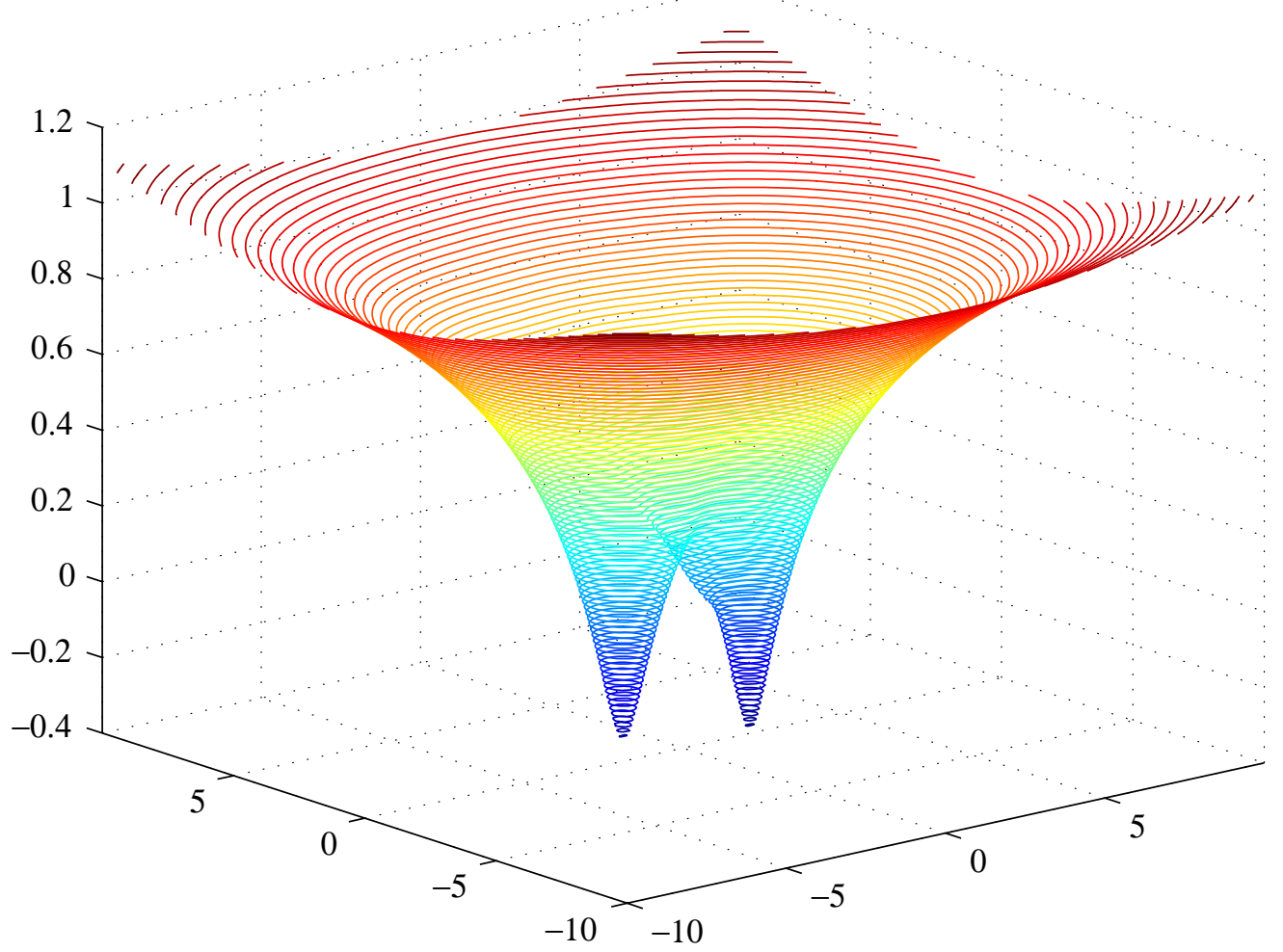

Figure 6: Contour plots of $\log _{10}\left(\sigma_{\min }([A-z I B])\right)$ for the pair (31) in 3D form. 


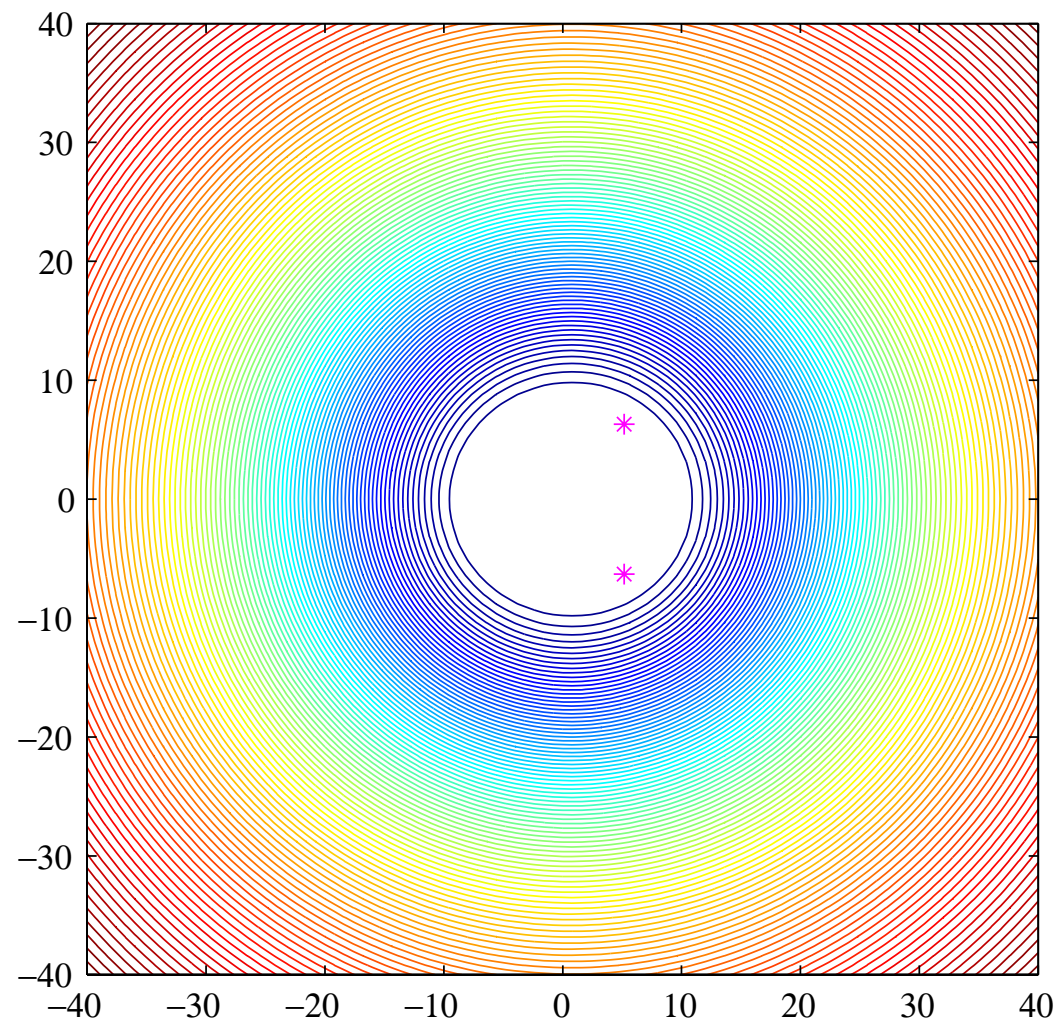

Figure 7: Contour plots of $\log _{10}\left(\sigma_{\min }([\widehat{A}-z I \widehat{B}])\right)$. The mark $*$ denote the location of the optimizer $z^{\star}=5.1740 \pm 6.3107 j$. 


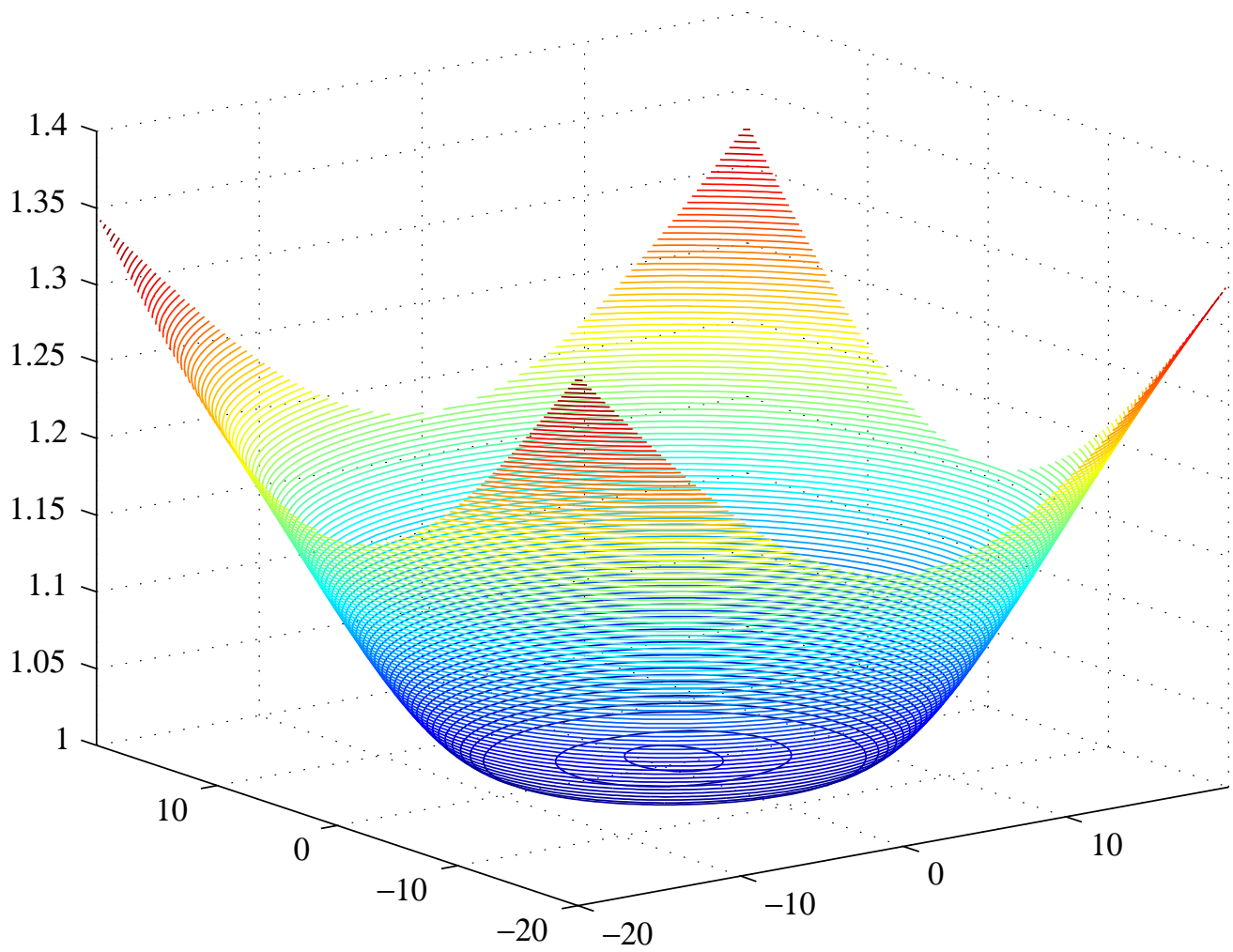

Figure 8: Contour plots of $\log _{10}\left(\sigma_{\min }([\widehat{A}-z I \widehat{B}])\right)$ in 3D form. 